\title{
Comparative Study of Feature Extraction Components from Several Wavelet Transformations for Ornamental Plants
}

\author{
Kohei Arai, Indra Nugraha Abdullah, Hiroshi Okumura \\ Graduate School of Science and Engineering \\ Saga University \\ Saga City, Japan
}

\begin{abstract}
Human has a duty to preserve the nature, preserving the plant is one of the examples. This research emphasis on ornamental plant that has functionality not only as ornament plant but also as a medicinal plant. Purpose of this research is to find the best of the particular feature extraction components from several wavelet transformations. It consists of Daubechies, Dyadic, and Dualtree complex wavelet transformation. Dyadic and Dual-tree complex wavelet transformations have shift invariant property. While Daubechies is a standard wavelet transform that widely used for many applications. This comparison is utilizing leaf image datasets from ornamental plants. From the experiments, obtained that best classification performance attained by Dual-tree complex wavelet transformation with $96.66 \%$ of overall performance result.
\end{abstract}

Keywords-wavelet transformation; shift invariant; rotation invariant; feature extraction; leaf identification.

\section{INTRODUCTION}

Oxygen is an essential part for all living things in the world. Plant plays an important role to produce oxygen and supply it for their sustainable life. The cycle between human and the plant is the interesting one. Carbon dioxide as the output of human respiratory is needed by plant for photosynthesis activity. Then, this activity is resulting oxygen which vital for human.

According to this cycle, human supposed to preserve the plant to maintain availability of oxygen. Based on International Union for Conservation of Nature and Natural Resources, the number of identified plant species which consist of Mosses, Ferns and Allies, Gymnosperms, Flowering Plants, Green Algae, Red Algae is about 307.674 species. [1].

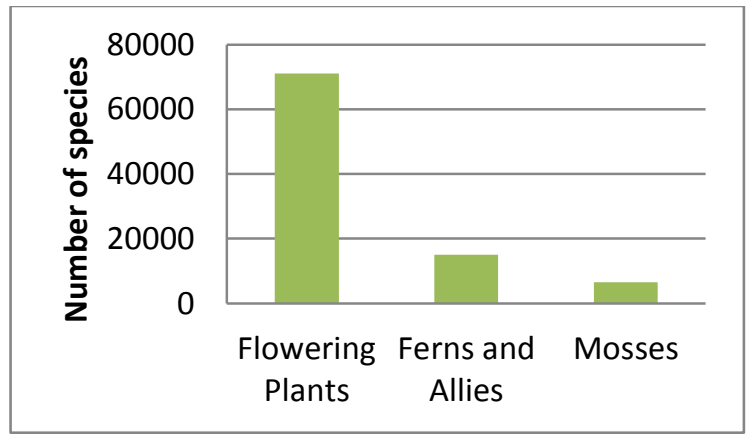

Fig. 1. Column charts number of unidentified plant species
On the other side, the approximate number of unidentified species is 80.500 species. It consists of Flowering Plants with 71.000 species, Ferns and Ales with 15.000 species, Mosses with 6.500 species [2]. Considering the highest number possessed by Flowering Plants, identification of the plants, which include also ornamental plant, has become a challenge for us.

As a recognition step for unidentified species, this research emphasis on ornamental plant, which has medicinal function. However, only few people know about its function as a treatment of the disease. As in Indonesia, this ornamental plant is mostly cultivated in front of the house. If this easiness and its medicinal function are taken into consideration, this plant should be an initial treatment or option towards full chemicalbased medicines. In 2005, Gu et al. proposed leaf recognition based on the combination of Haar discrete wavelet transformation and Gaussian interpolation [3]. They used the wavelet to make a leaf skeleton and the extract the feature using run-length feature. Followed by Casanova et al. in 2009, conducted a research with title plant leaf identification using Gabour wavelets [4]. They were used 20 Brazilian plant species, then identified using Gabour wavelets and compared it with Fourier descriptor and co-occurrences matrices.

This research is a sub-research of the main research. The main research is identification ornamental plant based on its color, shape, and texture information. In this study, we want to investigate the best of the particular feature extraction components, which had previously been conducted [5]. For this measurement process, we utilize several wavelet transformations. Start from Daubechies wavelet transformation, and Dyadic wavelet transformation which are the previous researches [6], and then Dual-tree wavelet transformation which is current main focus. Because of there are small numbers of papers dealing with utilization of wavelet transformation for leaf identification. We propose to do comparative study utilizing aforementioned wavelet transformations.

This paper is organized as follows. Section 2 will describe ornamental plant that used in this research. Follow by the next section that explains about the proposed method. Experiments results in section 3 . In the end of this paper, there are conclusion and future work. 


\section{ORNAMENTAL PLANTS}

Ornamental plant in this research is not general the ornamental plant, because besides has function as an ornamental plant, also can be used as herbal medicine to cure many diseases. The main focus of this research is to recognize this kind of plant from its leaf.

Image data set of ornamental leaf in this research was obtained from direct acquisition using digital camera. This data set is taken based on tropical ornamental plant that usually cultivated in front of the house in Indonesia. This data set contains 8 classes with 15 images for each class. The classes are Bay (syzygium polyanthum), Cananga (canagium odoratum, lamk), Mangkokan (nothopanax scutellarium merr.), Jasmine (jasminum sambac [soland]), Cocor bebek (kalanchoe pinnuta), Vinca (catharanthus roseus), Kestuba (euphorbia pulcherrima, willd), Gardenia (gardenia augusta, merr). In order to avoid expensiveness of computation, size of the image is 256 x 256 pixels. The sample images of each class are presented as follow:

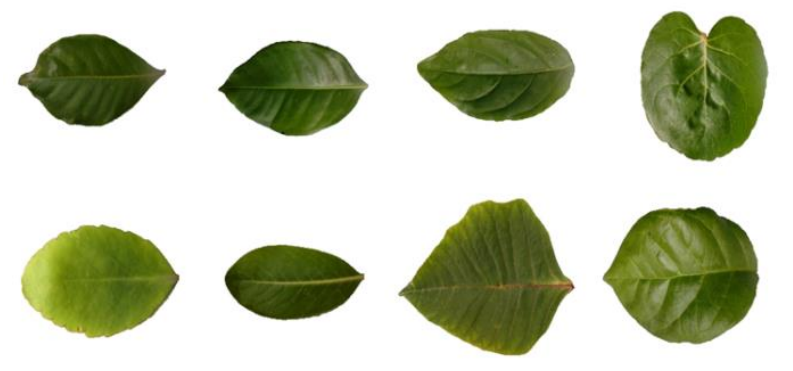

Fig. 2. Sample Images from every class inside data set

The Table 1 shows specific medicinal functions of the corresponding ornamental leaf. In Indonesia, there are two mostly used serving ways of these ornamental leaves. Firstly, boils the leaf together with water and apply as drinks. Secondly, put the leaf to the skin surface [7].

TABLE I. MEDICINAL FUNCTION OF ORNAMENTAL LEAF

\begin{tabular}{|l|c|}
\hline \multicolumn{1}{|c|}{ Name } & Medicinal Function \\
\hline Bay & Diarrhea, scabies and itching \\
\hline Cananga & Asthma \\
\hline Mangkokan & Mastitis, skin injury, hair loss \\
\hline Jasmine & Fever, head ache, sore eyes \\
\hline Cocor Bebek & Ulcer, diarrhea, gastritis \\
\hline Vinca & Diabetes, fever, burn \\
\hline Kestuba & Bruise, irregular menstrual \\
\hline Gardenia & Sprue, fever, constipation \\
\hline
\end{tabular}

\section{PROPOSED METHOD}

\section{A. Wavelet Transformations}

\section{1) Daubechies Wavelet Transformation}

For $N \in \mathbb{N}$, Daubechies wavelet of class D- $2 N$ is function $\psi={ }_{N} \psi \in L^{2}(R)$ denoted by

$$
\psi(x):=\sqrt{2} \sum_{k=0}^{2 N-1}(-1)^{k} h_{2 N-1-k} \varphi(2 x-k),
$$

where $h_{0}, \ldots, h_{2 N-1} \in R$ are the constant filter coefficients that fulfilling the conditions

$$
\sum_{k=0}^{N-1} h_{2 k}=\frac{1}{\sqrt{2}}=\sum_{k=0}^{N-1} h_{2 k+1}
$$

similarly, for $l=0,1, \ldots, N-1$,

$$
\sum_{k=2 l}^{2 N-1+2 l} h_{k} h_{k-2 l}= \begin{cases}1 & \text { if } l=0 \\ 0 & \text { if } l \neq 0\end{cases}
$$

and where $\varphi={ }_{N} \varphi: R \rightarrow R$ is the scaling function, given by the recursive equation

$$
\varphi(x)=\sqrt{2} \sum_{k=0}^{2 N-1} h_{k} \varphi(2 x-k)
$$

Daubechies orthogonal wavelets of classes D2 - D20 (only even index numbers) are the wavelets that generally used [8]. The index number belongs to the number $2 \mathrm{~N}$ of coefficient. Single wavelet has a number of vanishing moments equal to half the number of coefficients. In this study we propose to use Daubechies D4 wavelets (DB D4), it has two vanishing moments. With these vanishing moments D4 can encodes polynomial of two coefficients, for example constant and linear signal components.

\section{2) Dyadic Wavelet Transformation}

The downsampling wavelet, which samples the scale and translation parameters, is often fails when deal with some assignments such as edge detection, features extraction, and image denoising $[9,10]$. Different with the downsampling wavelet, the dyadic wavelet samples only the scale parameter of a continuous wavelet transform, and does not samples the translation factor. In one side, it creates highly redundant signal representation, but the other side, since it has shift-invariance ability, this method is a convincing candidate as a feature descriptor method.

Let $L^{2}(R)$ be the space of square integrable functions on real line $R$, and define the Fourier transform of the function $\psi \in L^{2}(R)$ by

$$
\hat{\psi}(\omega)=\int_{-\infty}^{\infty} \psi(t) e^{-i \omega t} d t
$$


If there is exist $A>0$ and $B$ such that

$$
A \leq \sum_{-\infty}^{\infty}\left|\hat{\psi}\left(2^{j} \omega\right)\right|^{2} \leq B
$$

then $\psi(t)$ is called dyadic wavelet function. Dyadic wavelet transform of $f(t)$ is defined using this $\psi(t)$ by

$$
W f\left(u, 2^{j}\right)=\int_{-\infty}^{\infty} f(t) \frac{1}{\sqrt{2^{j}}} \psi\left(\frac{t-u}{2^{j}}\right),
$$

from (6), $\hat{\psi}(0)=0$ must be satisfied, i.e., $\int_{-\infty}^{\infty} \psi(t) d t=0$. In order to design the dyadic wavelet function, we need a scaling function $\phi(t)$ satisfying a two-scale relation

$$
\phi(t)=\sum_{k} h[k] \sqrt{2} \phi(2 t-k)
$$

The scaling function $\phi(t)$ is usually normalized as $\int_{-\infty}^{\infty} \phi(t) d t=1$.

By (8), the Fourier transform of the scaling function resulting

$$
\hat{\phi}(\omega)=\frac{1}{\sqrt{2}} \hat{h}\left(\frac{\omega}{2}\right) \hat{\phi}\left(\frac{\omega}{2}\right),
$$

where $\hat{h}$ denotes a discrete Fourier transform

$$
\hat{h}(\omega)=\sum_{k} h[k] e^{-i \omega t}
$$

Since $\hat{\phi}(0)=1$, we can apply (9) and (10) to obtain $\hat{h}(0)=\sqrt{2}$. Using the scaling function and the wavelet filter $g[k]$, we define a dyadic function by $\psi(t)=\sum_{k} g[k] \sqrt{2} \phi(2 t-k)$.

The Fourier transform of $\psi(t)$,

$$
\hat{\psi}(\omega)=\frac{1}{\sqrt{2}} \hat{g}\left(\frac{\omega}{2}\right) \hat{\phi}\left(\frac{\omega}{2}\right)
$$

will be needed later.

Let us denote the discrete Fourier transform of the filters $h[k], h[k], \tilde{h}[k]$, and $\tilde{g}[k]$, by $h(\omega), h(\omega), \hat{\hat{h}}(\omega)$ and $\hat{\tilde{g}}(\omega)$ respectively.
We suppose that these Fourier transforms satisfy the below condition

$$
\widehat{\hat{h}}(\omega) \hat{h}^{*}(\omega)+\hat{\tilde{g}}(\omega) \hat{g}(\omega)=2, \quad \omega \in[-\pi, \pi],
$$

where * denotes complex conjugation. This condition called a reconstruction condition.

Under condition (12), we have

$$
a_{j+1}[n]=\sum_{k} h[k] a_{j}\left[n+2^{j} k\right] ; j=0,1, \ldots
$$

$$
d_{j+1}[n]=\sum_{k} g[k] a_{j}\left[n+2^{j} k\right] ; j=0,1, \ldots,
$$

here $a_{0}[n]$ is given by $a_{0}[n]=\int_{-\infty}^{\infty} f(t) \phi(t-n) d t$.

The (13) and (14) are dyadic decomposition formula for one-dimensional signals.

\section{3) Dual-tree Complex Wavelet Transformation}

Problems such as lack of shift invariances and poor directional selectivity that usually appears in DWT can be solved effectively using Dual-tree Complex Wavelet Transformation (DTCWT). Besides that ability, DTCWT also has limited redundancy and efficient order-N computation $[12,13]$.

By doubling the sampling rate at each level of the tree, we can obtain approximate shift invariance with real DWT. This doubling process is done by eliminating the downsampling by 2 after level 1, and this is equal with two parallel fullydecimated trees. The filters in one tree must supply half a sample different of delays from the other tree. Odd-length in one tree and even-length in the other are required for linear phase. The image below is the dual-tree filters for the CWT.

From Fig. 3, tree A is real DWT that gives real part of wavelet transform, and tree B is real DWT that gives imaginary part. These real DWTs use different sets of filters. $h 0, h l$ and g0, gl denote low-pass/high-pass filter pair for upper filter bank and low-pass/high-pass filter bank for lower filter bank, respectively.

Different with the real DWT which only have three sub images in total. DTCWT decomposes three sub images for each spectral quadrant 1 and 2 and will have six bandpass sub images of complex coefficient at each level. Because of the complex wavelet filters have the ability to separate positive from negative frequency vertically and horizontally, the orientation for these six sub images will cover $\pm 75^{0}, \pm 45^{0}, \pm 15^{0}$. 


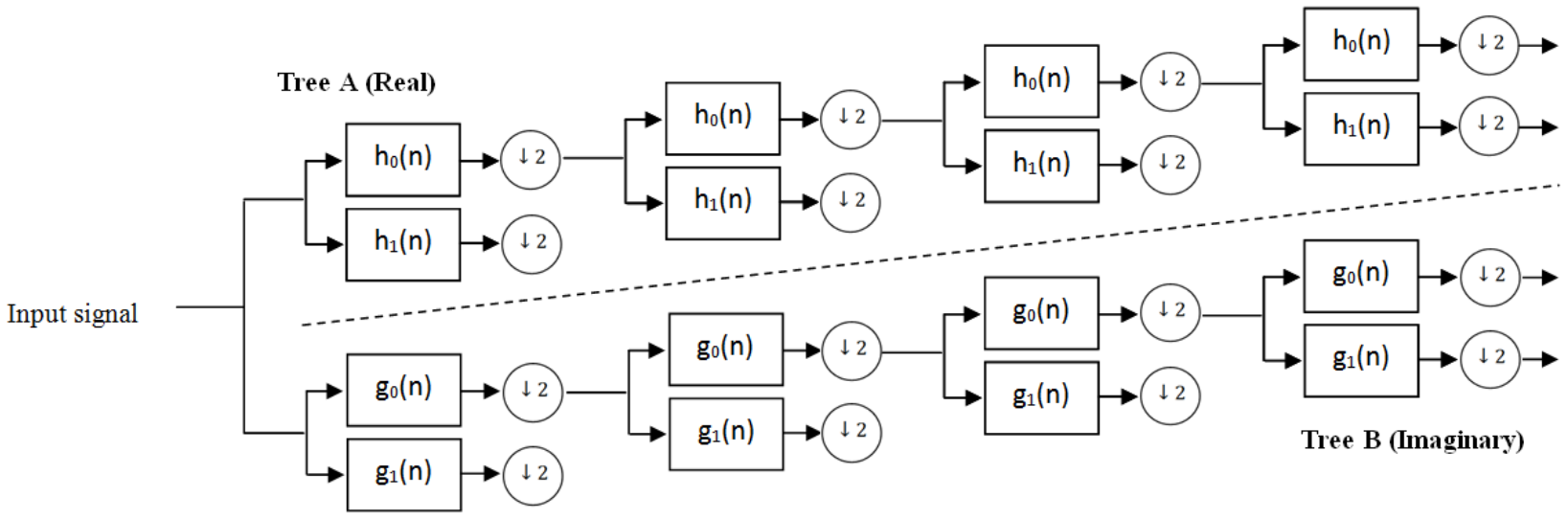

Fig. 3. Dual-tree filters for complex wavelet transformation

The following equation describes the detail:

$$
\begin{aligned}
& \psi_{i}(a, b)=\frac{1}{\sqrt{2}}\left(\psi_{1, i}(a, b)-\psi_{2, i}(a, b)\right), \\
& \left.\psi_{i+3}(a, b)=\frac{1}{\sqrt{2}} \psi_{1, i}(a, b)+\psi_{2, i}(a, b)\right)
\end{aligned}
$$

for $i=1,2,3, a$ denotes row, $b$ denotes column, definition of two separable 2-D wavelet bases are below:

$$
\begin{aligned}
& \psi_{1,1}(a, b)=\phi_{h}(a) \psi_{h}(b), \\
& \psi_{1,2}(a, b)=\psi_{h}(a) \phi_{h}(b), \\
& \psi_{1,3}(a, b)=\psi_{h}(a) \psi_{h}(b), \\
& \psi_{2,1}(a, b)=\phi_{g}(a) \psi_{g}(b), \\
& \psi_{2,2}(a, b)=\psi_{g}(a) \phi_{g}(b), \\
& \psi_{2,3}(a, b)=\psi_{g}(a) \psi_{g}(b)
\end{aligned}
$$

The purpose of utilization $1 / \sqrt{2}$ is to constitute an orthonormal operation from the sum/difference operation. Through the number of advantages from this DTCWT, we are expecting tight competition of performance with the Dyadic wavelet transformation.

\section{B. SVM Classifier}

SVM is a powerful tool for data classification. The indicators are the easiness to apply and impose Structural Risk Minimization (SRM). SRM armed the SVM to have strong ability in generalization of data. Its function is to minimize an upper bound on the expected risk. In principle, SVM learns to obtain optimal boundary with maximum margin that able to separate set of objects with different class of membership.

In order to achieve the maximum margin classifier, we have two options. Hard margin and soft margin are the options that totally depend on linearity of the data. Hard margin SVM is applicable to a linearly separable data set. However, often the data is not linearly separable. Soft margin SVM emerged as its solution [14,15]. The optimization problem for the soft margin SVM presented as below:

$$
\begin{gathered}
\min _{w, b} \frac{1}{2}\|w\|^{2}+C \sum_{i=1}^{n} \xi_{i} \\
\text { subject to: } y_{i}\left(w^{T} x_{i}+b\right) \geq 1-\xi_{i}, \quad \xi_{i} \geq 0 .
\end{gathered}
$$

where $w, C, \xi, b$ are the weight vectors, the penalty of misclassification or margin errors, the margin error, the bias, respectively.

In (18) can lead us to an efficient kernel methods approach. A kernel method is an algorithm that depends on the data only through kernel function, which computes a dot product in some possibly high dimensional data. Using the function $\phi$ training vector, the input space $x$ is mapped into higher dimensional space. $K\left(x_{i}, x_{j}\right)=\phi\left(x_{i}\right)^{T} \phi\left(x_{j}\right)$ is called kernel function. The degree of the polynomial kernel can control the flexibility of resulting classifier [15]. It will be appropriate with this research when we classify 8 classes of leaf. Polynomial kernel is shown in equation (19).

$$
K\left(x_{i}, x_{j}\right)=\left(\gamma x_{i}^{T} x_{j}+r\right)^{d}, \gamma>0 \text {. }
$$

Where $\gamma, r, d$ are kernel parameters, and $i, j$ denote $i^{t h}, j^{t h}$ vector in data set.

In this research, we propose to use Sequential Minimal Optimization (SMO). SMO act as efficient solver of the optimization problem in the training of support vector machines. SMO also solves the problems analytically by way of breaks the problems into a series of smallest possible problems.

\section{Feature Extraction Components}

In this research, we propose to use particular feature extraction components that extracted from the above wavelet transformations. The components are energy, mean, standard deviation and coefficient of variation. These components' values are extracted from four sub images, which are approximation, vertical, horizontal and diagonal sub images for DB D4 and Dyadic wavelet transformations. For DTCWT, those values are obtained from six sub images in real parts tree 
and six sub images in imaginary parts tree, in total is 12 sub images.

Because wavelet works in the frequency domain, energy is very useful as a feature extraction component. Energy values from several directions and different decomposition levels possibly well-capture the leaf main information which is leaf venation. The other component is mean value, and it can be interpreted as central of tendency of the ornamental leaf data. When standard deviation or predictable dispersion increases in proportion to concentration, we have the other value as a solution called coefficient of variation. Through these comprehensive and fully-related values, we believe that we can gain satisfying results.

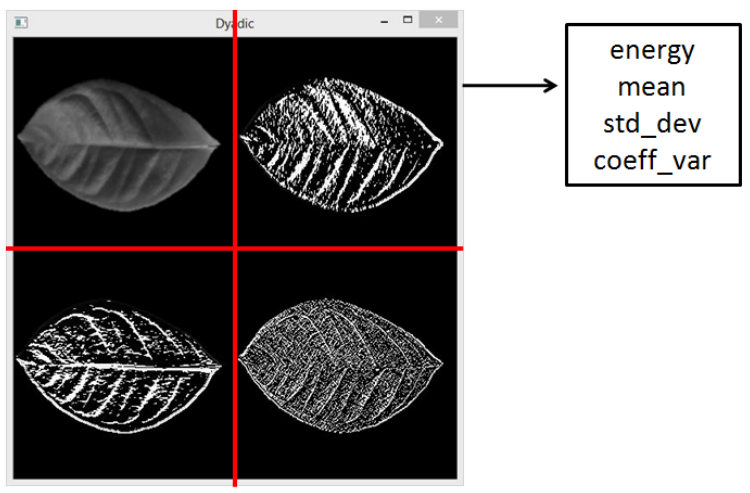

Fig. 4. Feature extraction components for all wavelet transformation

\section{EXPERIMENTS}

\section{A. Test Aspects}

Test aspects of this research consist of original (no change at all), rotation, scaling, translation, and perspective change. It means we have 5 different datasets with its corresponding test aspect. Inside the rotated dataset, there are images with dissimilar degrees of rotation start from $45^{\circ}, 90^{\circ}, 135^{\circ}, 180^{\circ}$, $225^{\circ}, 270^{\circ}$, and $315^{\circ}$. For scaled dataset, we have $30 \%$ and $60 \%$ downscaled images. Inside the translated dataset, we translated the images to many directions within the original image size. The last is perspective changed dataset with two different perspective changed, are applied to the images.

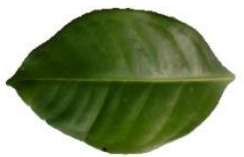

(a)

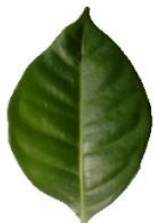

(b)

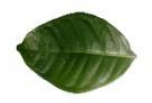

(c)



(e)

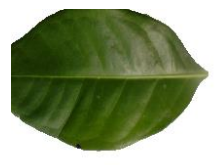

(d)
Fig. 5. Test aspects of ornamental leaf images. (a) original, (b) rotation, (c) scaling, (d) translation, (e) perspective change.

\section{B. Comparison Through Classification}

TABLE II. BEST DECOMPOSITION LEVEL FOR DB D4

\begin{tabular}{|c|c|c|c|}
\hline $\begin{array}{c}\text { Decomposition } \\
\text { level }\end{array}$ & Level 1 & Level 2 & Level 3 \\
\hline $\begin{array}{c}\text { Original } \\
\text { Dataset }\end{array}$ & $\mathbf{9 5 . 8 3}$ & 94.17 & 87.5 \\
\hline
\end{tabular}

TABLE III. BEST DECOMPOSITION LEVEL FOR DYADIC

\begin{tabular}{|c|c|c|c|}
\hline $\begin{array}{c}\text { Decomposition } \\
\text { level }\end{array}$ & Level 1 & Level 2 & Level 3 \\
\hline $\begin{array}{c}\text { Original } \\
\text { Dataset }\end{array}$ & $\mathbf{9 8 . 3 3}$ & 97.5 & 96.67 \\
\hline
\end{tabular}

TABLE IV. BEST DECOMPOSITION LEVEL FOR DUAL-TREE CWT

\begin{tabular}{|c|c|c|c|}
\hline $\begin{array}{c}\text { Decomposition } \\
\text { level }\end{array}$ & Level 1 & Level 2 & Level 3 \\
\hline $\begin{array}{c}\text { Original } \\
\text { Dataset }\end{array}$ & 95.83 & 96.67 & $\mathbf{9 9 . 1 7}$ \\
\hline
\end{tabular}

According to the result presented in Table 2 and Table 3, it was obtained that decomposition level 1 is the preferable result comparing to the other levels. Then, the best decomposition level for DTCWT was level 3. The reason for those preferable results are from that level was these wavelet transformation could extract very well the leaf main information called leaf venation, in comparison with the other level. 
TABLE V. COMPARISON USING VARIOUS DATASETS

\begin{tabular}{|c|c|c|c|c|c|c|}
\hline Datasets & Original & Translation & Rotation & Scaling & Perspective & Average \\
\hline DB D4 & 95.83 & 87.5 & 87.5 & 90.83 & 91.67 & 90.66 \\
\hline Dyadic & 98.33 & $\mathbf{9 7 . 5}$ & 94.17 & $\mathbf{9 6 . 6 7}$ & 95 & 96.33 \\
\hline Dual-tree CWT & $\mathbf{9 9 . 1 7}$ & 95.83 & $\mathbf{9 6 . 6 7}$ & 95.83 & $\mathbf{9 5 . 8 3}$ & $\mathbf{9 6 . 6 6}$ \\
\hline
\end{tabular}

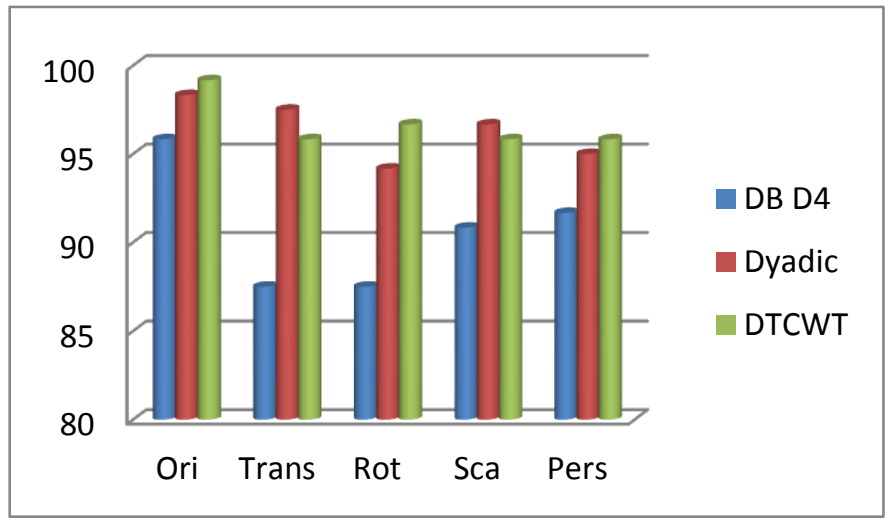

Fig. 6. Comparison of above mentioned feature extraction components using various wavelet transformations.

In Table 5 and Fig. 6 are presented the performance results of previously mentioned test aspects from wavelet transformations. For overall results, DB D4 gave us poor performance in all aspects. As expected, the tight competition came from the Dyadic wavelet transformation and DTCWT. For translation and scaling aspect, the dyadic wavelet transformation is superior to the others. Since sampling only scale parameter of continuous wavelet transformation, made the Dyadic has finer shift invariant property. Because of that reason also, the decomposition image in Dyadic will be exactly same with the input image, and feature extraction components in downscaled image that represent the leaf venation information still could capture.

However, for original, rotation and perspective, the DTCWT is superior. DTCWT has advantages in shift invariant and rotation invariant. Shift invariant property in the DTWCT is not perfect shift invariant but only nearly shift invariant. That reason made the Dyadic results more preferable in the translation dataset.

In the rotation aspect, DTCWT showed its advantages, through the directional selectivity support for six directions in real parts tree and six directions in imaginary parts tree, this DTCWT could give proper results. In original and perspective aspects, the only reason we gained superior results is the effectiveness of utilization 12 feature extraction components. Dissimilar with the DB D4 and Dyadic we only could utilize components from three directions of wavelet decomposition sub images plus one approximation image.

\section{CONCLUSIONS}

In this research, we have already measured the performance of Daubechies D4, Dyadic, and Dual-tree wavelet transformations. Daubechies D4 included in real DWT, which has several minus points, from the shift variance until lack of directional selectivity. Different with Daubechies, Dyadic and DTCWT has solutions to solve that shift variance problem. Because of the Dyadic sampling only scale parameter of continuous wavelet transformation, resulting highly redundant signals, but this is the important point when we try to extract information from the leaf for the translation and scaling aspects. Besides possess the nearly shift invariant property, DTCWT also support directional selectivity through six directions for real and imaginary parts, make them is superior for original, rotation and perspective aspect, as well as the overall result.

\section{FUTURE WORKS}

Hence the results from DTCWT outperform in comparison with the others. We still have a deficiency in translation and scaling aspects. Therefore, to find the best in all aspects we plan to conduct the comparative study involving the Double Density wavelet transformation and Double Density Dual-tree Complex wavelet transformation. Double Density wavelet transform has property nearly equal with the DTWCT, and the Double Density Dual-tree is the combination between both.

\section{REFERENCES}

[1] IUCN 2012. Numbers of threatened species by major groups of organisms (1996-2012) <http://www.iucnredlist.org/documents/summarystatistics/2012_2_RL_ Stats_Table_1.pdf >. Downloaded on 21 June 2013.

[2] Chapman A. D., "Numbers of living species in Australia and the World". Australian Government, Department of the Environment, Water, Heritage, and the Arts. Canberra, Australia, 2009.

[3] Casanova, D., de Mesquita Sá Junior, J. J. and Bruno, O. M., "Plant leaf identification using Gabor wavelets," Int. J. Imaging Syst. Technol., vol. 19, pp. 236-243, 2009.

[4] D.S. Huang, X.-P. Zhang, G.-B. Huang, "Leaf recognition based on the combination of wavelet Transform and gaussian Interpolation", ICIC 2005, Part I, LNCS 3644, pp. 253-262, 2005.

[5] Arai K., Abdullah I. N., Okumura H. and Kawakami R., "Improvement of automated detection method for clustered microcalcification based on wavelet transformation and support vector machine," International Journal of Advanced Research in Artificial Intelligence(IJARAI), 2(4), 2013.

[6] Arai K., Abdullah I. N., Okumura H., "Image identification based on shape and color descriptors and its application to ornamental leaf", IJIGSP, vol.5, no.10, pp.1-8, 2013. 
[7] Hartati Sri, "Tanaman hias berkhasiat obat. IPB Press," 2011. (In Indonesian)

[8] De Vries Andreas, "Wavelets", FH Sudwestfalen University of Applied Sciences, Hagen, Germany, 2006.

[9] Abdukirim T., Nijima K., Takano S. "Lifting dyadic wavelets for denoising," Proceedings of the Third International Workshop on Spectral Methods and Multirate Signal Processing (SMMSP), pp. 147154. 2003.

[10] Minamoto T., Tsuruta K., Fujii S. "Edge-preserving image denoising method based on dyadic lifting schemes," IPSJ Transactions on Computer Vision and Applications, vol. 2, 48-58, 2010.

[11] Mallat S., "A wavelet tour of signal processing," Academic Press. 1998.
[12] Kingsbury, N. G., "The dual tree complex wavelet transform: a new efficient tool for image Restoration and enhancement," In Proc. EUSIPCO98, Rhodes, Greece, 319-322, 1998.

[13] Selesnick I.W., Baraniuk R.G., and Kingsbury N.G., "The dual-tree complex wavelet transformation", IEEE Signal Processing Magazine, pp. 123-151, November 2015.

[14] Ben-Hur Asa, Weston Jason, “A user's guide to support vector machine. Data Mining Techniques for the Life Science", 223-239, Humana Press, 2010.

[15] Hsu Chih-Wei, Chang Chih-Chuang, Lin Chih-Jen, "A practical guide to support vector classification," Department of Computer Science National Taiwan University, Taiwan, 2010.

[16] Starck Jean-Luc, Murtagh Fionn, Fadili Jalal M. Sparse image and signal processing. Cambridge University Press. 2010. 